\title{
Efforts to induce cardiac electrophysiological properties in skeletal myoblasts in vitro
}

\author{
Xaver Koenig1', Eva Zebedin-Brandl ${ }^{1}$, Markus Milleㄹ, Michael Schnürch², \\ Moumita Koley ${ }^{2}$, Marko D Mihovilovic ${ }^{2}$, Rene Cervenka ${ }^{1}$, Hannes Todt ${ }^{1}$ and \\ Karlheinz Hilber*1
}

\author{
Address: ${ }^{1}$ Institute of Pharmacology, Center for Biomolecular Medicine and Pharmacology, Medical University of Vienna, 1090 Vienna, Austria \\ and 2Institute of Applied Synthetic Chemistry, Vienna University of Technology, 1060 Vienna, Austria \\ Email: Karlheinz Hilber* - karlheinz.hilber@meduniwien.ac.at \\ * Corresponding author
}

from 14th Scientific Symposium of the Austrian Pharmacological Society (APHAR)

Innsbruck, Austria. 21-22 November 2008

Published: 5 November 2008

BMC Pharmacology 2008, 8(Suppl I):A4I doi:I0.| |86/|47|-22I0-8-SI-A4|

This abstract is available from: http://www.biomedcentral.com/|47|-22I0/8/SI/A4I

(c) 2008 Koenig et al; licensee BioMed Central Ltd.

\section{Background}

When the myocardium is injured by an acute infarction, a fibrous, non-contractile scar develops, because mature cardiac tissue cannot effectively regenerate. In patients this often results in congestive heart failure, one of the major health problems in the developed world. Although multipotent cardiac stem cells, which could support myocardial regeneration, were recently identified, their limited availability prevents therapeutic applications. More readily available stem cell populations derived from other tissues, such as undifferentiated skeletal myocytes (myoblasts) or bone marrow-derived adult stem cells, have been shown to be capable of repairing cardiac damage in animal models. These cell types, however, have a very limited capacity to transdifferentiate into functional cardiomyocytes after transplantation into the heart. This fact certainly hampers their beneficial therapeutic effects. A strategy to overcome this problem would be the induction of cardiomyogenic function in stem cells prior to transplantation. Here, we tried two different in vitro strategies to achieve this goal in skeletal myoblasts.

\section{Materials and methods}

Mouse C2C12 skeletal myoblasts were incubated in differentiation media preconditioned by primary cardiomyocyte cultures (cardiac preconditioning), or treated with the cardiomyogenic small molecule cardiogenol C for 2-14 days. Thereafter, their electrophysiological properties were measured using the whole cell patch clamp technique, and compared with those of untreated control cells.

\section{Results}

Simulation of a cardiac cell environment by "cardiac preconditioning" altered the $\mathrm{Na}^{+}$current activation and inactivation properties of $\mathrm{C} 2 \mathrm{C} 12$ cells from skeletal muscle to more cardiac-like ones. Tetrodotoxin and RT-PCR experiments suggest that an upregulation of the expression of the cardiac sodium channel isoform $\mathrm{Na}_{\mathrm{V}} 1.5$ versus the skeletal muscle isoform $\mathrm{Na}_{\mathrm{V}} 1.4$ is responsible for the observed changes in sodium current function. Similar effects on the $\mathrm{Na}^{+}$currents of $\mathrm{C} 2 \mathrm{C} 12$ cells were observed in cells treated with the cardiomyogenic small molecule cardiogenol C.

\section{Conclusion}

We conclude that cardiac preconditioning and treatment with cardiogenol $\mathrm{C}$ affect the electrophysiological properties of skeletal myoblasts. Such strategies may be useful to generate myoblasts with more cardiac-like electrophysiological properties.

\section{Acknowledgements}

Support: Austrian Science Fund PI9352-BII. 